\section{Spasmolytic Cocktail of Nitroglycerin and Verapamil versus Verapamil Alone for the Prevention of Radial Artery Spasm (RAS)}

\author{
Cassidy Harmon ${ }^{1 *}$, Marintha Short ${ }^{1}$ and Nezar Falluji ${ }^{2}$ \\ ${ }^{1}$ Pharmacy, Saint Joseph Hospital, Lexington, KY USA \\ ${ }^{2}$ Cardiology, Saint Joseph Hospital, Lexington, KY USA
}

\begin{abstract}
A major potential complication when utilizing transradial access for coronary procedures is Radial Artery Spasm (RAS). To minimize the risk of RAS, intra-arterial vasodilators are used to maximize the diameter of the artery and prevent injury to the endothelium that may cause it to spasm. No published data verifies whether combination therapy has an advantage over a single agent for this indication. This single-center non-concurrent study (historical cohort study) of patients evaluated the use of verapamil alone versus the cocktail of verapamil and nitroglycerin for the prevention of RAS. There was no difference observed in the primary outcome, incidence of RAS, in the nitroglycerin and verapamil group (4.5\%) versus the verapamil alone group $(9.5 \%, p=0.589)$. Changes in blood pressure and heart rate as well as change to femoral access, procedural success, and local forearm pain were similar between the two groups. Using verapamil alone for the prevention of RAS may be as effective and safe as using a cocktail of nitroglycerin and verapamil. Larger studies are needed to confirm this conclusion.
\end{abstract}

Keywords: Nitroglycerin; Radial artery spasm; RAS; Vasospasm; Verapamil

\section{Introduction}

A popular alternative to the traditional transfemoral access for coronary catheterizations is utilizing the radial artery as a point of access. With a transradial approach, the risk for bleeding is significantly diminished. If the radial artery becomes occluded through thrombosis or traumatic injury, the ulnar artery will still supply collateral blood flow. Also, there are no major structures

${ }^{*}$ Corresponding author: Cassidy Harmon, Pharmacy, Saint Joseph Hospital, 1 Saint Joseph Drive, Lexington, KY USA, Tel: +1 5134062428; Fax: +1 8593132974; E-mail: cmharm2@gmail.com

Citation: Harmon C, Short M, Falluji N (2016) Spasmolytic Cocktail of Nitroglycerin and Verapamil versus Verapamil Alone for the Prevention of Radial Artery Spasm (RAS). J Cardiol Stud Res 3: 010.

Received: January 08, 2016; Accepted: February 09, 2016; Published: February 23, 2016

Copyright: (c) 2016 Harmon C, et al. This is an open-access article distributed under the terms of the Creative Commons Attribution License, which permits unrestricted use, distribution, and reproduction in any medium, provided the original author and source are credited. surrounding that radial artery that can be damaged through this access site [1].

Transradial access for these procedures has become increasingly popular as it is associated with lower vascular complication rates, earlier patient mobilization, less patient discomfort, and lower procedural costs compared to the femoral approach [2].

The radial artery is made up of smooth muscle cells in the tunica media that are stimulated by circulating catecholamines and by mechanical stimuli including vessel cannulation and manipulation of the catheter or guidewire. When these muscle cells are stimulated, it causes a tonic contraction of the smooth muscle and a reduction in the vessel's diameter, leading to one of the most significant complications with transradial access, Radial Artery Spasm (RAS) [2]. RAS is defined as a temporary narrowing of the radial artery causing difficulty in manipulation of the catheter. It is aggravated by movement of the catheter or sheath and usually causes pain in the patient's forearm [3]. This is one of the most common complications in radial Percutaneous Coronary Interventions (PCIs), seen in up to $22 \%$ of patients who utilize this transradial approach without the use of intra-arterial vasodilators. RAS is associated with procedural failure [2]. During RAS, the vessel clamps down on the guidewire, making it difficult to advance the sheath or guidewire within the vessel, often resulting in failure to cannulate the vessel. This resistance during manipulation can cause significant pain to the patient.

Certain risk factors have been identified as predictors of RAS. These include: a small baseline radial artery diameter or tortuosity of the vessel, a low outer sheath diameter to radial artery diameter ratio, longer and more complicated procedures, a high number of exchanged catheters, unsuccessful first attempts at cannulation, female sex, shorter wrist circumference, inexperienced physician performing the procedure, and younger age $[4,5]$. Utilizing sheaths and catheters with hydrophilic coatings, which allow them to easily slide into the vessel without stimulating the vessel wall, have been shown in studies to reduce the incidence of spasm $[3,5,6]$. Longer sheaths protect the whole vessel but have been associated with a higher incidence of spasm [3,6,7].

Administration of heparin can through the arterial sheath can also aggravate spasm. Radial artery occlusion can occur if the radial artery has a small diameter relative to the sheath or guidewire and the patient experiences RAS, further decreasing the diameter of the vessel. Therefore, it is extremely important to utilize various approaches to minimize the risk of RAS, including the use of intra-arterial vasodilators to maximize the diameter of the artery. Maximizing the diameter of the artery can help prevent injury to the endothelium that may cause it to spasm [8].

Several combinations of medications, specifically vasodilators, have been given intra-arterially for prevention of RAS in procedures using transradial access. The SPASM 1 and 2 trials evaluated the use of verapamil $2.5 \mathrm{mg}$ with molsidomine $1 \mathrm{mg}$, a long-acting nitric oxide releasing vasodilator, and showed that the combination of these two agents reduced the incidence of RAS compared to placebo [9]. The most commonly used cocktails consist of nitroglycerin and a calcium-channel blocker, but it is unclear whether combination therapy with nitroglycerin and verapamil has an advantage over the single agent verapamil. In a trial by Kiemeneij et al., verapamil $5 \mathrm{mg}$ 
and nitroglycerin $200 \mathrm{mcg}$ decreased the incidence of RAS from $22 \%$ with placebo to $8 \%$ [2]. Most of these combinations are advocated based off of provider experience. The combination of nitroglycerin and verapamil is a commonly used effective combination in the prevention of RAS [2]. However, single agents are used as well. Intravenous nitroglycerin has historically been unattainable at times due to manufacturer backorder, and verapamil as a single agent has shown to reduce the incidence of RAS.

Verapamil is a calcium-channel blocker that causes dilation of the coronary and systemic arteries, including the radial arteries, through blocking the influx of extracellular calcium ions in cardiac and vascular smooth muscles. Compared with the calcium-channel blocker diltiazem, verapamil has been shown to be more effective at prevention of RAS. The SPASM3 study compared patients receiving diltiazem $5 \mathrm{mg}$, verapamil $2.5 \mathrm{mg}$ and Isosorbide Dinitrate (ISDN) $1 \mathrm{mg}$ and showed a considerable reduction in RAS with verapamil and with ISDN compared to diltiazem [3]. This study aims to evaluate the use of verapamil alone versus the cocktail of verapamil and nitroglycerin for the prevention of RAS in patients undergoing cardiac procedures through a transradial approach.

\section{Materials and Methods}

This is a single-center non-concurrent study (historical cohort study) conducted at a 433-bed tertiary care center looking at the use of verapamil alone versus a combination of verapamil and nitroglycerin for the prevention of RAS in patients undergoing cardiac procedures utilizing a transradial approach. Procedures were performed by one interventional cardiologist with extensive experience using the radial approach (annual workload of radial cases is approximately 90).

Two arms of patients were analyzed from January 1, 2014 to February 9, 2015. The first arm included 67 patients who received the cocktail of nitroglycerin $300 \mathrm{mcg}$ and verapamil $2.5 \mathrm{mg}$, administered in separate syringes to preserve stability, for the prevention of RAS. The second arm included 21 patients who received verapamil $2.5 \mathrm{mg}$ alone for the prevention of RAS. Each regimen was given in addition to 3,000 IU of unfractionated heparin in the radial artery after sheath insertion. (See figure 1, Patient Population). Patients were included if they were at least 18 years of age and undergoing a cardiac catheterization by transradial approach, even if they had undergone previous transradial catheterization. Patients were excluded if they were unable to receive one of the study medications (nitroglycerin or verapamil) due to drug allergies or alternate reasons.

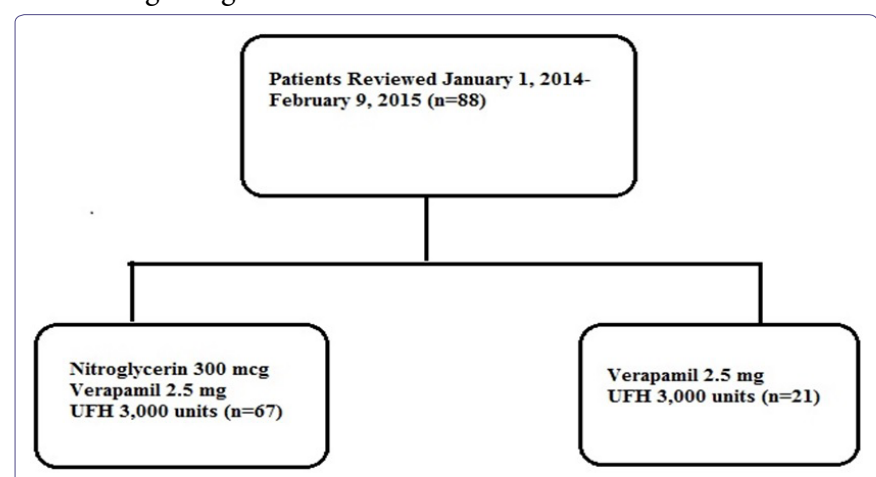

Figure 1: Patient population.

*UFH: Unfractionated Heparin

The primary endpoint was occurrence of RAS while the secondary endpoints were changes in blood pressure and heart rate, procedural success, and change to femoral access. The occurrence of RAS was defined as significant difficulty with insertion, manipulation, and/or withdrawal of the sheath or catheter along with pain perceived by the patient. This outcome was dictated in the patient's cardiac catheterization operative note if experience by the patient. Procedural success was defined as the completion of the intended procedure at the initial access site without major complications (death, acute myocardial infarction, coronary bypass surgery) or significant residual stenosis. Total procedure time was defined as the time from the local anesthetic injection to the introducer sheath removal. Changes in heart rate and blood pressure were measured as the largest changes from baseline to after administration of the intra-arterial vasodilators.

\section{Statistical Analysis}

Data was analyzed using Microsoft Excel. Continuous data was analyzed with student t-test. Categorical or nominal data was analyzed with chi-squared or Fisher's exact tests when any of the sample size subgroups were less than or equal to five. All tests used $95 \%$ confidence interval with an $\alpha$ of less than 0.05 . To achieve an $80 \%$ power to detect a $20 \%$ difference between the two groups, 27 patients were needed in each group. This study did not meet power.

\section{Results}

From January 1, 2014 to February 9, 2015, a total of 88 patients were reviewed and included in this study. There were 67 patients who received a combination of nitroglycerin and verapamil for prevention of RAS and 21 patients who received verapamil alone (see figure 1, patient population). Outcomes measured included incidence RAS, procedural success, crossover to femoral artery, and changes in heart rate and blood pressure. This data was collected from electronic patient charts.

Baseline characteristics were similar between the two groups of patients receiving cardiac catheterizations (see table 1, baseline demographics). A higher percentage of males were seen in the group that received verapamil alone. This is significant because there is a greater incidence of RAS in women than men, potentially due to a

\begin{tabular}{|c|c|c|c|}
\hline & $\begin{array}{c}\text { Nitroglycerin+Verapamil, } \\
\mathbf{n = 6 7}(\%)\end{array}$ & $\begin{array}{c}\text { Verapamil, n=21 } \\
\mathbf{( \% )}\end{array}$ & P-value \\
\hline Male Gender & $35(52.2)$ & $17(81.0)$ & 0.023 \\
\hline White Race & $63(94.0)$ & $18(85.7)$ & 0.35 \\
\hline Procedure Type & & & \\
\hline Diagnostic only & 47 & $17(81.0)$ & 0.409 \\
\hline With intervention & $20(30.0)$ & $4(19.0)$ & 0.409 \\
\hline Hyperlipidemia & $55(82.1)$ & $15(71.4)$ & 0.354 \\
\hline Hypertension & $61(91.0)$ & $18(85.7)$ & 0.680 \\
\hline Diabetes & $27(40.3)$ & $9(42.9)$ & 0.835 \\
\hline Smoking Status & & & \\
\hline Never smoker & $20(29.9)$ & $6(33.3)$ & 0.763 \\
\hline Current smoker & $36(53.7)$ & $8(38.1)$ & 0.035 \\
\hline Former smoker & $11(16.4)$ & $4(19.0)$ & 0.241 \\
\hline EF $\leq 40 \%$ & $6(9.0)$ & $19(90.5)$ & 0.144 \\
\hline $\begin{array}{c}5 F r \text { Sheath } \\
\text { (versus 6Fr) }\end{array}$ & $50(74.6)$ & & 0.044 \\
\hline
\end{tabular}

EF: Ejection Fraction

*Note: no patients in this study had an $E F<20 \%$ 


\begin{tabular}{|c|c|c|c|}
\hline & $\begin{array}{c}\text { Nitroglycerin+Verapamil, } \\
\mathbf{n = 6 7}(\mathbf{S D})\end{array}$ & $\begin{array}{c}\text { Verapamil, } \mathbf{n = 2 1} \\
\text { (SD) }\end{array}$ & P-value \\
\hline Age, y & $57.5(13.40)$ & $62.2(13.76)$ & 0.166 \\
\hline Height, cm & $170.2(10.01)$ & $175.0(7.63)$ & 0.048 \\
\hline Weight, kg & $90.7(28.26)$ & $97.8(25.91)$ & 0.315 \\
\hline BMI & $30.9(8.02)$ & $31.9(8.21)$ & 0.636 \\
\hline $\begin{array}{c}\text { Procedural Time, } \\
\text { min }\end{array}$ & $30.7(18.25)$ & $29.3(13.17)$ & 0.748 \\
\hline Diagnostic & $21.7(8.35)$ & $26.4(11.43)$ & 0.132 \\
\hline Interventional & $52.8(16.13)$ & $46.0(7.35)$ & 0.436 \\
\hline \multicolumn{4}{|c|}{ Table 1: Baseline demographics. } \\
\hline
\end{tabular}

BMI: Body Mass Index; SD: Standard Deviation

shorter wrist circumference. Patients were around 60 years old and $20-30 \%$ of them had an interventional cardiac catheterization. The majority of patients were white, had hypertension and hyperlipidemia, and were either current or former smokers. The sheath size used (mainly $5 \mathrm{Fr}$ ) and the procedural time (approximately 30 minutes) were both similar between the two groups.

There was no significant difference in the primary endpoint, the incidence of RAS, between the two groups. Three patients (4.5\%) experience RAS in the group of patients who received nitroglycerin and verapamil $(n=67)$ and two patients $(9.5 \%)$ experienced RAS in the group of patients who received verapamil alone $(\mathrm{p}=0.589)$. (See table 2, Incidence of RAS). This outcome was dictated in each of the patients' operative notes by the physician who performed the cardiac catheterization. Of the patients who experienced RAS, four were female with an average age of 56.2 years, which is younger than the total average age. Two underwent an intervention rather than simply a diagnostic catheterization. All of them had both hypertension and hyperlipidemia and were current smokers. The average procedure time was 38.6 minutes, which was longer than the average procedural time of the total population.

\begin{tabular}{|c|c|c|c|}
\hline & $\begin{array}{c}\text { Nitroglycerin+Verapamil, } \\
\mathbf{n = 6 7}(\%)\end{array}$ & $\begin{array}{c}\text { Verapamil, } \mathbf{n}=\mathbf{2 1} \\
(\%)\end{array}$ & P-value \\
\hline $\begin{array}{c}\text { Radial artery } \\
\text { spasm }\end{array}$ & $3(4.5)$ & $2(9.5)$ & 0.589 \\
\hline \multicolumn{4}{|c|}{ Table 2: Incidence of RAS. } \\
\hline
\end{tabular}

Documentation in the electronic medical record was less consistent with secondary efficacy outcomes (see table 3, secondary efficacy outcomes). Rates of procedural success were high at around $90 \%$. There were no differences observed in the incidence of

\begin{tabular}{|c|c|c|c|}
\hline Outcome & $\begin{array}{c}\text { Nitroglycerin+Verapamil, } \\
\mathbf{n = 6 7}(\%)\end{array}$ & $\begin{array}{c}\text { Verapamil, } \mathbf{n = 2 1} \\
\mathbf{( \% )}\end{array}$ & P-value \\
\hline $\begin{array}{c}\text { Moved to an al- } \\
\text { ternate position }\end{array}$ & $7(10.4)$ & $1(4.8)$ & 0.674 \\
\hline $\begin{array}{c}\text { Changed to } \\
\text { femoral access }\end{array}$ & $2(3.0)$ & $1(4.8)$ & 1.000 \\
\hline $\begin{array}{c}\text { Aborted proce- } \\
\text { dure }\end{array}$ & $58(86.6)$ & $19(90.5)$ & 0.729 \\
\hline $\begin{array}{c}\text { Procedural } \\
\text { success }\end{array}$ & $11(16.4)$ & $2(9.5)$ & 0.512 \\
\hline $\begin{array}{c}\text { Resistance } \\
\text { during maneuver }\end{array}$ & $4(6.0)$ & $0(0.0)$ & 0.569 \\
\hline $\begin{array}{c}\text { Local forearm } \\
\text { pain }\end{array}$ & \multicolumn{2}{|c|}{ Table 3: Secondary efficacy outcomes. } \\
\hline
\end{tabular}

procedural access that was changed to the femoral site or abortion of the procedure. The rates of resistance during maneuver of the catheter, and documented local forearm pain were very low and not significantly different in those who received verapamil and nitroglycerin versus verapamil alone.

Administration of intra-arterial vasodilators was well-tolerated among both groups of patients. Blood pressure and heart rate changes were similar between the two groups of patients undergoing cardiac catheterization. There were no incidences of severe systemic hypotension or bradycardia requiring intervention such as fluids or vasopressor support. The changes in systolic and diastolic blood pressure and heart rate were not significantly different between the two groups. (See table 4, secondary safety outcomes). Concomitant medications potentially causing changes in heart rate or blood pressure were not analyzed.

\begin{tabular}{|c|c|c|c|}
\hline Outcome & $\begin{array}{c}\text { Nitroglycerin+Verapamil, } \\
\mathbf{n = 6 7}(\mathrm{SD})\end{array}$ & $\begin{array}{c}\text { Verapamil, } \mathbf{n = 2 1} \\
\text { (SD) }\end{array}$ & P-value \\
\hline $\begin{array}{c}\text { Change in SBP, } \\
\text { mean (SD), } \\
\mathrm{mmHg}\end{array}$ & $28.5(17.08)$ & $23.9(18.03)$ & 0.296 \\
\hline $\begin{array}{c}\text { Change in DBP, } \\
\text { mean (SD), } \\
\mathrm{mmHg}\end{array}$ & $16.4(11.82)$ & $11.2(11.61)$ & 0.084 \\
\hline $\begin{array}{c}\text { Change in HR, } \\
\text { mean (SD), BPM }\end{array}$ & $3.6(14.87)$ & $2.5(5.96)$ & 0.742 \\
\hline \multicolumn{3}{|c|}{ Table 4: Secondary safety outcomes. } \\
\hline
\end{tabular}

DBP: Diastolic Blood Pressure; HR: Heart Rate; SBP: Systolic Blood Pressure

\section{Discussion}

Centers performing cardiac catheterizations using a radial approach utilize a variety of intra-arterial vasodilators for prevention of RAS. Currently, a popular approach is to use a cocktail of intra-arterial verapamil and nitroglycerin for this indication. In an era when medication shortages are common and cost-savings is vital, it is prudent to evaluate the efficacy of combination therapy with nitroglycerin and verapamil and compare that to using verapamil alone for prevention of RAS in radial cardiac catheterizations. Although no studies have compared the combination therapy to verapamil alone, verapamil is used as a single agent for prevention of RAS by many interventional cardiologists. This is the first study to compare the cocktail of nitroglycerin and verapamil to verapamil alone for the prevention of RAS.

This study did not find a difference in the incidence of RAS between patients who underwent cardiac catheterizations utilizing a radial approach receiving nitroglycerin and verapamil versus verapamil alone. In the present study, most of the patients who experienced RAS were female and had a longer procedural time, which is consistent with some of the well-known risk factors for RAS. However, there was a significantly higher percentage of males in the group of patients who received verapamil alone than those who received verapamil with nitroglycerin, which could have skewed results.

This study was limited by its retrospective nature and small sample sizes. However, other major clinical trials evaluating vasodilator use for prevention of RAS, such as SPASM3, also did not meet power [3]. Another major limitation was the incompleteness in documentation, particularly with the secondary efficacy endpoints. For example, pain scales were not recorded in operative notes and alternative adverse effects of administration of these agents such as rash, itching, and 
burning sensation in the forearm were not recorded. Because this was a retrospective analysis, investigators were limited by information dictated in operative notes, which may not have been complete.

Many patients undergoing these cardiac catheterizations received medications other than the specified dose of intra-arterial vasodilators that could have impacted the patients' vital signs and other outcomes. These medications included morphine, benzodiazepines, and extra boluses of the vasodilators. This data was not collected and analyzed. The secondary outcomes of resistance during catheter maneuver and local forearm pain were not always associated with RAS, although causes of these outcomes were not recorded.

Strengths of this trial include a lack of provider bias. A single interventional cardiologist was used in this analysis, removing the concern for varied skill sets from multiple providers. This cardiologist provided dictated operative notes along with a note that detailed exact times when doses of medications were given and vital signs were read as well as notes about what was happening during the procedure. These details were recorded in real time by a technician in the catheterization laboratory, making most of the documentation for these procedures incredibly thorough.

Although no clinical trials have compared the efficacy of the cocktail of nitroglycerin and verapamil to verapamil alone for prevention of RAS, this trial provides some evidence that it may be appropriate to use verapamil alone. Particularly in times of intravenous nitroglycerin manufacturer shortages, this may provide an appropriate alternative for facilities using combination therapy, especially in patients without risk factors for RAS such as smaller baseline radial artery diameter, female gender, and more complicated procedures utilizing a higher number of exchanged catheters or multiple attempts at cannulation.

\section{Conclusion}

Using intra-arterial verapamil alone for the prevention of RAS is as effective and safe as using a cocktail of intra-arterial nitroglycerin and verapamil. Larger studies are needed to confirm this conclusion. When using nitroglycerin is not an option to use in combination with verapamil for the prevention of RAS in the setting of a coronary procedure utilizing radial access, such as during times of medication shortages or drug allergies, verapamil alone may be a safe and effective alternative.

\section{Acknowledgements}

This research received no specific grant from any funding agency in the public, commercial, or not-for-profit sectors.

\section{Conflict of Interests}

The authors declare that there are no conflicts of interest.

\section{Research Ethics}

Catholic Health Initiatives Institute for Research and Innovation Institutional Review Board (CHIRB) has approved this research for exemption with a reference number of 716433-2.

\section{References}

1. Kiemeneij F, Laarman GJ, de Melker E (1995) Transradial artery coronary angioplasty. Am Heart J 129: 1-7.

2. Kiemeneij F, Vajifdar BU, Eccleshall SC, Laarman G, Slagboom T, et al. (2003) Evaluation of a spasmolytic cocktail to prevent radial artery spasm during coronary procedures. Catheter Cardiovasc Interv 58: 281-284.

3. Rosencher J, Chaïb A, Barbou F, Arnould MA, Huber A, et al. (2014) How to limit radial artery spasm during percutaneous coronary interventions: The spasmolytic agents to avoid spasm during transradial percutaneous coronary interventions (SPASM3) study. Catheter Cardiovasc Interv 84: 766-771.

4. Jia DA, Zhou YJ, Shi DM, Liu YY, Wang JL, et al. (2010) Incidence and predictors of radial artery spasm during transradial coronary angiography and intervention. Chin Med J (Engl) 123: 843-847.

5. Ruiz-Salmerón RJ, Mora R, Vélez-Gimón M, Ortiz J, Fernández C, et al (2005) [Radial artery spasm in transradial cardiac catheterization. assessment of factors related to its occurrence, and of its consequences during follow-up]. Rev Esp Cardiol 58: 504-511.

6. Koga S, Ikeda S, Futagawa K, Sonoda K, Yoshitake T, et al. (2004) The use of a hydrophilic-coated catheter during transradial cardiac catheterization is associated with a low incidence of radial artery spasm. Int J Cardiol 96: 255258.

7. Rathore S, Stables RH, Pauriah M, Hakeem A, Mills JD, et al. (2010) Impact of length and hydrophilic coating of the introducer sheath on radial artery spasm during transradial coronary intervention: a randomized study. JACC Cardiovasc Interv 3: 475-483.

8. Boyer N, Beyer A, Gupta V, Dehghani H, Hindnavis V, et al. (2013) The effects of intra-arterial vasodilators on radial artery size and spasm: implications for contemporary use of trans-radial access for coronary angiography and percutaneous coronary intervention. Cardiovasc Revasc Med 14: 321-324.

9. Varenne O, Jégou A, Cohen R, Empana JP, Salengro E, et al. (2006) Prevention of arterial spasm during percutaneous coronary interventions through radial artery: the SPASM study. Catheter Cardiovasc Interv 68: 231-235. 